\title{
An Experimental Validation of An Online Adaptive Cooperation Scheme for Spectrum Sensing
}

\author{
Serhan Yarkan*, B. Uğur Töreyin*, Khalid. A. Qaraqe* and A. Enis Çetin ${ }^{\S}$ \\ ${ }^{*}$ Electrical \& Computer Engineering, Texas A \& M University \\ $\S$ Dept. of Electrical and Electronics Engineering, Bilkent University \\ E-mails: syarkan@ece.tamu.edu,khalid.qaraqe@qatar.tamu.edu, \\ behcet.toreyin@qatar.tamu.edu, cetin@bilkent.edu.tr
}

\begin{abstract}
Cooperative spectrum sensing methods in the literature assume a static communication scenario with fixed channel and propagation environment characteristics. In order to maintain the level of sensing reliability and performance under changing channel and environment conditions, in this study, an online adaptive cooperation scheme is proposed. Energy detection data from each cooperating sensor are fused together by an adaptive weighted linear combination at the fusion center. Weight update operation is performed online through the use of orthogonal projections onto convex sets (POCS). Also, in this paper, an end-to-end methodology for a flexible experimental setup is proposed. This setup is specifically deployed to emulate the proposed adaptive cooperation scheme for spectrum sensing and validate its practical use in cognitive radio systems.

Index Terms-projection onto convex sets, cognitive radio systems, adaptive data fusion, online learning, experimental setup, wireless propagation
\end{abstract}

\section{INTRODUCTION}

Cognitive radio (CR) systems, which are aware of their surroundings and has the ability of self-adaptation to dynamic environmental and channel conditions, have emerged as a novel paradigm in wireless communications [1]. One of the most distinguished features of these systems is spectrum sensing for dynamic spectrum access. It has been reported repeatedly in the literature that cooperation among spectrum sensors substantially increases the overall performance and sensing reliability of a CR system $[2,3]$. Furthermore, cooperative spectrum sensing has been shown to mitigate the negative effects that stem from practical problems like multipath fading/shadowing and noise power fluctuations by exploiting the diversities among multiple CRs (spectrum sensors) [3].

The literature on cooperative spectrum sensing has been exponentially growing for the last couple of years [3]. However, existing methods are developed based on a static communication scenario assumption with fixed channel and radio frequency $(\mathrm{RF})$ propagation environment characteristics. It would be too optimistic to admit that these cooperative techniques, with their non-adaptive nature, can survive in practice under dynamically shifting channel and environmental conditions.

Online learning approaches are powerful tools for problems where drifts in concepts take place which are very much similar to the problems specifically encountered in non-stationary random surroundings of a practical CR system. In this study,

$$
\text { 978-1-4577-0024-8/11/\$26.00 (c) 2011 IEEE }
$$

an adaptive data fusion (ADF) scheme, which exploits inherent dynamics of the sensing problem by adapting the weight of the contribution from each spectrum sensor in an online manner, is proposed for cooperative spectrum sensing. Each sensor carries out conventional energy detection operation. Then, the output of energy detectors is sent to the fusion center for their corresponding weights to be updated online based on the ADF scheme through the use of orthogonal projections onto convex sets (POCS).

There is a vast literature on the system models suggested and their analyses for spectrum sensing. However, reports on practical implementations of such models are limited. It is obvious that practical considerations for an end-to-end communications system introduce many physical layer issues such as synchronizing and frequency-offset estimation and recovery, and some other receiver impairments, which are just three among many to name few. This shows that experimental validation of such theoretical models and analysis under practical considerations is key to measure the performance of the proposed models. Therefore, in this study, an end-to-end methodology for a flexible experimental setup is proposed. Even though the experimental setup is designated specially for the proposed online adaptive cooperation scheme, with the use of the proposed end-to-end methodology, many other system models involving different types of channels and environments can be validated with slight modifications in it.

In this regard, the contributions of this study can be summarized as: (I) An online adaptive cooperation scheme is proposed for the spectrum sensing problem. This way, the level of sensing reliability and performance of sensing operation is maintained. (II) In addition, an end-to-end methodology for a flexible experimental setup is proposed. This setup is specifically deployed to emulate the proposed adaptive cooperation scheme for spectrum sensing and validate its practical use in cognitive radio systems. However, as shown in the sequel, proposed methodology and experimental setup may be implemented with slight modifications to investigate other system models developed for different practical scenarios.

The organization of the paper is as follows. The statement of the problem and the system model are presented in Section II. In Section III, the proposed online adaptation and data fusion method is described. Details of the experimental setup, data collection, and processing are given in Section IV. Numerical results and discussions are presented in Section V. Finally, 
conclusions are drawn in the last section.

\section{Statement of the Problem And System Model}

Let a fixed, immobile spectrum sensor network be composed of $M$-many sensors, possibly situated at $M$ geographically different locations. Each sensor, say $\operatorname{Sensor}_{i}$, where $i=$ $1, \ldots, M$, carries out a sequence of analysis steps proposed in [4] upon receiving a signal $r_{i}(\cdot)$ as shown in Figure 1. The baseband equivalent of each one of the received signal by the $i$-th sensor, that is $r_{i}(\cdot)$, can be represented as:

$$
r_{i}(t)=\left\{\begin{array}{rr}
v_{i}(t), & H_{0}, \\
x(t)+\quad v_{i}(t), & H_{1},
\end{array}\right.
$$

where $v_{i}(t)$ is complex additive white Gaussian noise $(\mathrm{AWGN})$ with $\mathcal{C N}\left(0, \sigma_{i}^{2}\right)$ in the form of $v_{i}(t)=v_{I, i}(t)+$ $j v_{Q, i}(t)$ as both $v_{I, i}(t)$ and $v_{Q, i}(t)$ being $\mathcal{N}\left(0, \sigma_{i}^{2} / 2\right)$ and $j=\sqrt{-1} ; x(t)$ is the complex baseband equivalent of the unknown signal transmitted by a single mobile source; $H_{0}$ represents the hypothesis corresponding to absence of the unknown signal, whereas $H_{1}$ refers to the hypothesis corresponding to presence of it at time $t$. Then, the problem can be stated as identifying the absence/presence of the unknown signal, $x(t)$, by taking into account dynamic changes in the channel and the propagation environment that possibly stem from such practical problems and physical phenomena as shadowing, fading, mobility, etc.

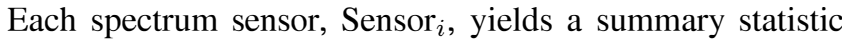
$u_{i}$, where $i=1, \ldots, M$. At this point, it is worth mentioning that the output of each sensor can be considered to be of discrete form rather than a continuous form such as $u_{i}[n]$ for the sake of notational convenience in the subsequent steps. Summary statistic at $n$-th discrete time instant, $u_{i}[n]$ is calculated by each secondary user over a detection interval of $N$ samples as follows:

$$
u_{i}[n]=\sum_{k=n-N+1}^{n}\left|r_{i}[k]\right|^{2} \quad i=1,2, \ldots, M
$$

These data are transmitted over a very narrow-band channel, say "Broadcast Fusion Channel," to an immobile "fusion center." In this regard, since sensors and fusion center are all assumed to be fixed, the set of signals reaches at the fusion center at $n$-th discrete time instant can be modeled as a Gaussian channel with zero-mean noise $q_{i}$ and with variance $\delta=\left[\delta_{1}^{2}, \delta_{2}^{2}, \ldots, \delta_{M}^{2}\right]^{T}$ :

$$
\mathbf{y}[n]=\mathbf{u}[n]+\mathbf{q}[n]
$$

with $\mathbf{y}[n]=\left[y_{1}[n], \ldots, y_{M}[n]\right], \mathbf{u}[n]=\left[u_{1}[n], \ldots, u_{M}[n]\right]$ and $\mathbf{q}[n]=\left[q_{1}[n], \ldots, q_{M}[n]\right]$. At the fusion center, where the adaptive data fusion is realized online, a global test statistic $y_{c}[n]$ is computed from $y_{i}[n]$. Carrying out analysis steps similar to the one presented in [2], the following linear rule at the fusion center with a test threshold $\gamma_{c}[n]$ is obtained:

$$
y_{c}[n] \gtreqless \mathcal{H}_{1} \gamma_{c}[n]
$$

where

$$
y_{c}[n]=\sum_{i=1}^{M} w_{i}[n] y_{i}[n]=\mathbf{y}^{T}[n] \mathbf{w}[n]
$$

and

$$
\mathbf{w}[n]=\left[w_{1}[n], \ldots, w_{M}[n]\right]^{T}, \quad w_{i}[n] \geq 0
$$

The performance metric used for this system model is the probability of false alarm $\left(P_{f}\right)$ and the probability of detection $\left(P_{d}\right)$ :

$$
P_{f}=Q\left[\frac{\gamma_{c}-N \sigma^{\mathbf{T}} \mathbf{w}}{\sqrt{\mathbf{w}^{\mathbf{T}} \mathbf{\Sigma}_{\mathcal{H}_{\mathbf{o}}} \mathbf{w}}}\right]
$$

and

$$
P_{d}=Q\left[\frac{\gamma_{c}-\left(N \sigma+\mathbf{E}_{\mathbf{s}} \mathbf{g}\right)^{\mathbf{T}} \mathbf{w}}{\sqrt{\mathbf{w}^{\mathbf{T}} \mathbf{\Sigma}_{\mathcal{H}_{\mathbf{1}}} \mathbf{w}}}\right]
$$

where $Q($.$) is the complementary cumulative distribution$ function, which calculates the tail probability of a zero mean unit variance Gaussian random variable, $\sigma=\left[\sigma_{1}, \ldots, \sigma_{\mathbf{M}}\right]$, $\boldsymbol{\Sigma}_{\mathcal{H}_{0}}$ and $\boldsymbol{\Sigma}_{\mathcal{H}_{1}}$ are system parameter matrix defined in [2]. For the sake of brevity, the sample number index, $n$, is removed in (7) and (8).

Note that, different weight selection rules can be employed for different purposes, such as the ones described in $[5,6]$. In this study, considering the dynamical characteristics of the mobile radio channel that undergoes shadowing, amplitude fading, and Doppler spread, as well as changes in propagation environment, an online adaptive data fusion method based on POCS is proposed for the combined test statistic $y_{c}[n]$ calculation. As a result, the spectrum sensing system as a whole, becomes capable of adapting itself to changes dynamically occuring in the channel and the propagation environment by tracking the second-order data output from individual spectrum sensors.

\section{PRoposed Method}

Let $\mathbf{w}[n]=\left[w_{1}[n], \ldots, w_{M}[n]\right]^{T}$ be the weight vector at each spectrum sensor at time step $n$. Then we define

$$
y_{c}[n]=\mathbf{y}^{\mathbf{T}}[n] \mathbf{w}[n]=\sum_{i} w_{i}[n] y_{i}[n]
$$

as an estimate of the test threshold, $\gamma_{c}[n]$, at time step $n$, and the error, $e_{c}[n]=\gamma_{c}[n]-y_{c}(n)$. For a fixed value of probability of false alarm, $P_{f}$, one can obtain the corresponding threshold value, $\gamma_{c}[n]$, from (7) as:

$$
\gamma_{c}=N \sigma^{\mathbf{T}} \mathbf{w}+\mathbf{Q}^{-\mathbf{1}}\left(\mathbf{P}_{\mathbf{f}}\right) \sqrt{\mathbf{w}^{\mathbf{T}} \boldsymbol{\Sigma}_{\mathcal{H}_{\mathbf{o}}} \mathbf{w}}
$$

At each time step $n, \gamma_{c}[n]$ is evaluated using (10), for a fixed $P_{f}$ value and the weight vector, w $[n]$. Along with the weight update equation presented in the next subsection, (10) provides weights adapt themselves in such a way that statistics of $P_{f}$ is not affected from dynamic changes and drifts in the channel and/or the propagation environment. This is achieved via the controlled feedback mechanism based on the error term, $e_{c}[n]$. One of the main advantages of the proposed online cooperative spectrum sensing strategy compared to other related methods in $[2,5-8]$, is this feedback mechanism. Weights of the spectrum sensors yielding correlation estimates different than (same as) the test threshold are reduced (increased) iteratively at each time step, making it possible to keep the performance of sensing not affected from the change in channel characteristics. Another advantage of the proposed algorithm is that it does not assume any specific probability distribution about the data. 


\section{A. Set Theoretic Weight Update Algorithm}

Ideally, weighted sum of the received summary statistics of spectrum sensors should be equal to the test threshold $\gamma_{c}[n]$ :

$$
\gamma_{c}[n]=\mathbf{y}^{T}[n] \mathbf{w}
$$

which represents a hyperplane in the M-dimensional space, $\mathbf{w} \in \mathbb{R}^{M}$. Hyperplanes are convex in $\mathbb{R}^{M}$. At time instant $n, \mathbf{y}^{T}[n] \mathbf{w}[n]$ may not be equal to $\gamma_{c}[n]$. The next set of weights are determined by projecting the current weight vector $\mathbf{w}[n]$ onto the hyperplane represented by (11). This process is geometrically depicted in Figure 6. The orthogonal projection $\mathbf{w}[n+1]$ of the vector of weights $\mathbf{w}[n] \in \mathbb{R}^{M}$ onto the hyperplane $\gamma_{c}[n]=\mathbf{y}^{T}[n] \mathbf{w}$ is the closest vector on the hyperplane to the vector $\mathbf{w}[n]$.

Let us formulate the problem as a minimization problem:

$$
\min _{\mathbf{w}^{*}}\left|\mathbf{w}^{*}-\mathbf{w}[n]\right|, \quad \text { subject to: } \mathbf{y}^{T}[n] \mathbf{w}^{*}=\gamma_{c}[n]
$$

Solution can be obtained by using Lagrange multipliers:

$$
\mathcal{L}=\sum_{i}\left(w_{i}[n]-w_{i}^{*}\right)^{2}+\lambda\left(\mathbf{y}^{T}[n] \mathbf{w}^{*}-\gamma_{c}[n]\right)
$$

Taking partial derivatives with respect to $w_{i}^{*}$ :

$$
\frac{\partial \mathcal{L}}{\partial w_{i}^{*}}=2\left(w_{i}[n]-w_{i}^{*}\right)+\lambda y_{i}[n], \quad i=1, \ldots, M,
$$

setting the result to zero:

$$
2\left(w_{i}[n]-w_{i}^{*}\right)+\lambda y_{i}[n]=0, \quad i=1, \ldots, M
$$

and defining the next set of weights as $\mathbf{w}[n+1]=\mathbf{w}^{*}$ a set of $M$ equations is obtained:

$$
\mathbf{w}[n+1]=\mathbf{w}[n]+\frac{\lambda}{2} \mathbf{y}[n]
$$

The Lagrange multiplier, $\lambda$, can be obtained from the condition equation:

$$
\mathbf{y}^{T}[n] \mathbf{w}^{*}-\gamma_{c}[n]=0
$$

as follows:

$$
\lambda=2 \frac{\gamma_{c}[n]-y_{c}[n]}{\|\mathbf{y}[n]\|^{2}}=2 \frac{e_{c}[n]}{\|\mathbf{y}[n]\|^{2}}
$$

where the error, $e_{c}[n]$, is defined as

$$
e_{c}[n]=\gamma_{c}[n]-y_{c}[n]
$$

and $y_{c}[n]=\mathbf{y}^{T}[n] \mathbf{w}[n]$. Plugging this into (16)

$$
\mathbf{w}[n+1]=\mathbf{w}[n]+\frac{e_{c}[n]}{\|\mathbf{y}[n]\|^{2}} \mathbf{y}[n]
$$

is obtained. Hence the projection vector is calculated according to (20).

Whenever a new set of summary statistics, $\mathbf{u}[n]$ are generated from spectrum sensors, another hyperplane is defined in $\mathbb{R}^{M}$ based on the new data values $\mathbf{y}[n]$ arrived at the fusion center from the broadcast fusion channel

$$
\gamma_{c}[n+1]=\mathbf{y}^{T}[n+1] \mathbf{w}^{*}
$$

This hyperplane will probably not be the same as $\gamma_{c}[n]=$ $\mathbf{y}^{T}[n] \mathbf{w}[n]$ hyperplane as shown in Figure 6. The next set of weights, $\mathbf{w}[n+2]$, are determined by projecting $\mathbf{w}[n+1]$ onto the hyperplane in (21). Iterated weights converge to the intersection of hyperplanes, $\mathbf{w}^{\mathbf{c}}$, [9].The rate of convergence can be adjusted by introducing a relaxation parameter $\mu$ to (20) as follows

$$
\mathbf{w}[n+1]=\mathbf{w}[n]+\mu \frac{e_{c}[n]}{\|\mathbf{y}[n]\|^{2}} \mathbf{y}[n]
$$

where $0<\mu<2$ should be satisfied to guarantee the convergence according to the POCS theory $[10,11]$.

If the intersection of hyperplanes is an empty set, then the updated weight vector simply satisfies the last hyperplane equation. In other words, it tracks test threshold, $\gamma_{c}[n]$, by assigning proper weights to individual spectrum sensors, in order to maintain the same $P_{f}$ value under dynamically changing channel and propagation environment characteristics. Note that, the proposed online method does not need to wait until convergence to make a decision.

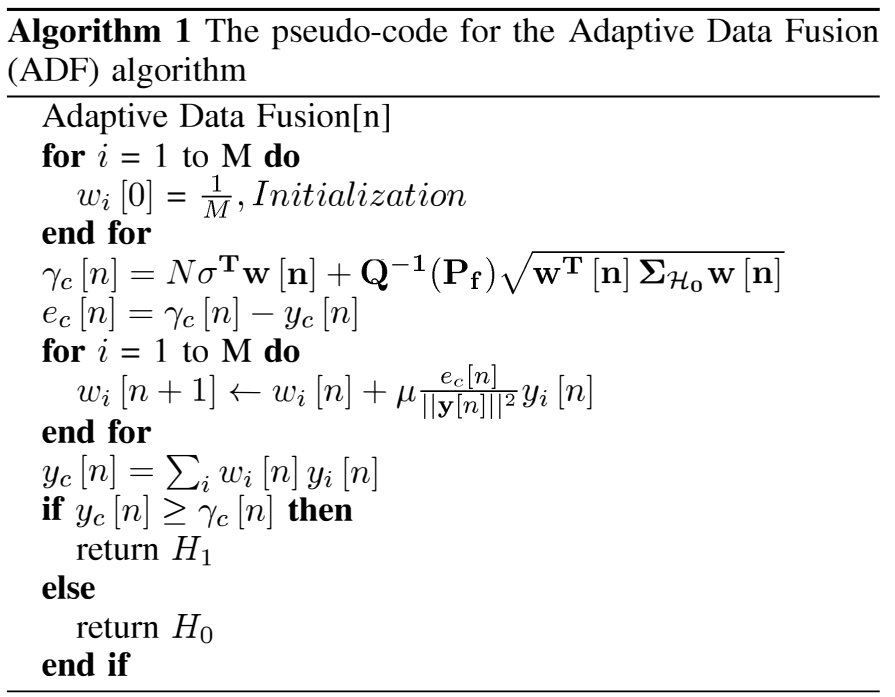

\section{Experimental Setup, Data Collection and PROCESSING}

The entire set of experiments and data collection were realized in Texas A\&M University at Qatar, Doha, at the Wireless Research Laboratory located in the Department of Electrical and Computer Engineering. The details of the experimental setup, devices and equipments used will be given in this section along with the methodology employed in data collection and processing.

\section{A. Experimental Setup, Devices, and Equipments Used}

As shown in Figure 1, there are two different types of channels in the the system model: (C.1) the channel between the unknown signal source and sensors, and (C.2) the channel between sensors and the ADF center, which is also referred to as broadcast fusion channel. In the system model, (C.1) denotes the channel representing a conventional wireless propagation environment through which sensors receive the signal. In the experiment, (C.1) is emulated by capturing the signal that is transmitted over the air by an unknown signal source under $H_{1}$ hypothesis.In contrast to (C.1), system model 
assumes an AWGN channel for (C.2). In order to emulate such a scenario a direct cable (wired) connection is used between transmitter-receiver pair. Characteristics of the emulated (C.2) will be discussed subsequently.

Since there are two different types of channels, there are two types of transmitter-receiver pairs as well operating on each side of both (C.1) and (C.2). In the experiments, Agilent vector signal generator (VSG) E4438C is used for emulating the transmitters in the system model as: (T.1) unknown signal source whose presence/absence will be identified, and (T.2) the sensors (energy detectors) that function as transmitters by sending their output after applying energy detection operation through the broadcast fusion channel. Since VSG E4438C can be programmed and controlled through the use of a computer and of a software running on it such as MATLAB, both types of transmitters (i.e., (T.1) and (T.2)) can be emulated by a single VSG in time division multiple access (TDMA) fashion.

In parallel with the transmitters, there are also two different types of receivers operating on each end of (C.1) and (C.2): (R.1) sensors, and (R.2) ADF center. In order to emulate both (R.1) and (R.2), Agilent PSA Series vector signal analyzer (VSA) E4440A is used.

In the sequel, it is worth mentioning some important characteristics of both VSG and VSA. Agilent VSG E4438C can be used as a transmitter since many important transmission parameters along with the baseband data to be transmitted can be controlled through the use of MATLAB. Beside its many built-in functions, the device allows to employ and modify very well-known standards such as Global System for Mobile (GSM) and North American Digital Cellular (NADC). Also, the authors developed a specific MATLAB script which can control and modify the transmit parameters of VSG and enables the VSG to transmit specific in-phase/quadrature (I/Q) values to act as a (T.2)-type transmitter. Similarly, Agilent PSA Series VSA E4440A is used as a receiver which yields baseband I/Q samples as its output for given reception settings. The VSA is controlled by its software interface that allows one to receive the signal and to provide the output as I/Q samples in vector format that can directly be used in MATLAB. Block diagram of the experimental setup is given in Figure 2 with corresponding identifiers described in Section IV-A.

\section{B. Experimental Methodology and Data Collection}

The experiment is realized in two stages as shown in Figure 2: (I) The part that includes (T.1)-(C.1)-(R.1) path and (II) the remaining part which includes (T.2)-(C.2)-(R.2) path. First stage is established and repeated for each sensor in a TDMA fashion and received baseband signal (the output of VSA) is fed into MATLAB. For $H_{0}$ hypothesis, of course, the unknown signal source emulated by VSG is inactive so that rx-side of each sensor (i.e., (R.1)) emulated by VSA captures the ambient noise over the air. For $H_{1}$ hypothesis, the VSG is programmed in such a way that it continuously transmits a random data (which exist in the memory of the device) according to NADC standard with the given transmit parameters. Data corresponding to each hypothesis are recorded for a very large period of time in order to obtain sufficient statistics. Recorded data are, later on, split into parts and organized in such a way that each part corresponds to an independent realization.
At the second stage of the experiment, upon splitting and organizing, the recorded data (baseband I/Q values) for each hypothesis are placed into the memory of the VSG through the use of MATLAB. This way, the tx-side of each sensor (i.e., (T.2)) is realized. Note that (C.2) is assumed to be AWGN in the system model mentioned earlier. Therefore, a direct cable connection is used between VSG and VSA to emulate the AWGN channel. Such a realization is reasonable due to the following observations. First of all, a perfectly isolated direct cable connection implies a frequency-flat channel response. Second, assuming that no changes occur in the experimental setup throughout the data collection, the power of the channel remains the same and acts as a fixed scaling factor (corresponding to cable loss) to the data transmitted. Third, even though the cable connection is assumed to be perfect, thermal noise, which exists in the RF front-end of the receiver and can be characterized as a disturbance of AWGN form, cannot be avoided and always exists in the received signal. These reasons show that a direct cable connection provides a reasonable approximation to realization of a theoretical AWGN channel. Parameters of the VSA are set to receive the data sent over the cable. When the transmission begins, VSA starts to capture the signal and store it on a computer to be fed into MATLAB on which $\mathrm{ADF}$ algorithm runs to generate the output.Finally, the output of $\mathrm{ADF}$ is obtained and results are shown and interpreted.

\section{Data Formatting and Processing}

At the second stage of experiment, upon capturing the data by VSA, a normalization is performed. Such a step might be required, especially when $H_{1}$ hypothesis is of concern, since the transmission loss causes the captured baseband I/Q values to be very small for VSG to deal with. The same reasoning is valid for $H_{0}$ assuming that the captured data is a bandlimited complex AWGN of $\mathcal{C N}(0,1)$. However, prior to this normalization step, one should consider the synchronization between VSG and VSA. Synchronization is extremely important, because data collection step for ADF to work properly relies on obtaining the correct values. Therefore, before transmitting the output of each sensor through the use of VSG, output of energy detectors are placed into the memory of VSG in the following format: $\mathbf{p}_{x+y}=\left(\begin{array}{l}\mathbf{0}_{x} \\ \mathbf{d}_{y}\end{array}\right)$, where $\mathbf{0}_{x}$ denotes the zero-padding operation with a zero-vector of length $x$ and $\mathbf{d}_{y}$ represent the output of the energy detector for any sensor, and $\mathbf{p}_{x+y}$ denotes the packet format to be sent over the cable through the use of VSG. Unless stopped, VSG keeps sending the information back to back; therefore, in order to make sure VSA captures at least one complete $\mathbf{d}$, capturing time of VSA is set to three times the total duration of a single packet $\mathbf{p}$. With the aid of the packet format $\mathbf{p}$ employed, burst detection is employed to synchronize the packet in which the data is placed. Next, again by taking advantage of $\mathbf{0}_{x}$ upon the burst detection, noise power estimation and normalization mentioned earlier in this subsection are established. Due to the clock mismatch between the VSG and VSA, a clock recovery algorithm is implemented to remove the frequency drift present in the I/Q samples that are provided by VSA. As will be shown subsequently, the direct cable connection (experimental broadcast fusion channel) would not exhibit a perfect AWGN 
behavior due to the inherent pulse shaping filter operation performed by VSG beside some other impairments such as coupling and so on. However, after the employment of burst detection, normalization, and clock recovery steps, one can assume that the output of broadcast fusion channel is obtained as close as possible to the theoretical model proposed. Finally, the real part of output data are fed into $\mathrm{ADF}$ in order to make a decision in regards to $H_{0}$ and $H_{1}$ hypotheses.

\section{RESULTS AND DISCUSSION}

Throughout the experiment, the sampling rate of VSA for both types of receivers (i.e., (R.1) and (R.2)) is chosen to be $\Delta t_{s}=10^{-5}$ s. In realizing $H_{1}$ hypothesis, a pseudo-random sequence is chosen in VSG memory to be continuously transmitted in NADC format to emulate the unknown signal source. For the first stage of the experiment, VSG is kept at a place where optical line-of-sight (LOS) is present between the VSG and VSA. Each sensor collects $N=20$ complex samples to generate an output for the energy detector and be fed into the broadcast fusion channel. For each sensor, this procedure is repeated 5000 times to obtain as many independent realizations as possible. In total, $M=20$ sensors are assumed to be present in the environment where all of them are assumed to have LOS with the unknown signal source.

After collecting the data for both hypotheses, ADF is fed by the experimental data in such a way that a Bernouilli random variable decides whether the input to ADF is selected from $H_{0}$ data or from $H_{1}$ data. Note that such a scheme constitutes one of the most challenging spectrum sensing behavior for ADF, since Bernoulli random variable implies maximum uncertainty about the absence/presence of the unknown signal. It must be stated here that most of the practical communication systems (depending also on the traffic type) transmit signals in bursts. Nevertheless, for the sake of testing the proposed method under challenging conditions, maximum uncertainty about the absence/presence of the unknown signal is adopted.

First of all, noise characteristics of both channels (C.1) and (C.2) should be investigated. Due to space limitations, only autocorrelation coefficient estimates of the captured data under $H_{0}$ hypothesis will be examined in comparison with those of pseudo-noise case. The results are plotted in Figure 3. As can be seen, autocorrelation estimates (unbiased) of the captured data exhibit significant correlation within the first $0.4 \mathrm{~ms}$. This mainly stems from VSA filter which introduces correlation and distorts the statistical characteristics of the noise.

When the processed data collected through the use of experimental setup, they are fed into the proposed ADF mechanism. The result is given in Figure 4. From Figure 4, one can conclude that ADF performs quite well under practical scenarios by solely looking at the $P_{m}=1-P_{d}$ values obtained. However, it is desired to investigate the theoretical results in a comparative manner to draw a solid conclusion regarding the performance of the ADF under practical conditions. For this purpose, the proposed ADF algorithm is examined for the following two cases. In the first case, ADF algorithm running on the receiver is assumed to be fed directly by the output of broadcast fusion channel (i.e., (C.2)). In other words, in the first case the baseband I/Q samples generated by (R.2) are the exact same values as those of the output of (C.2). In the

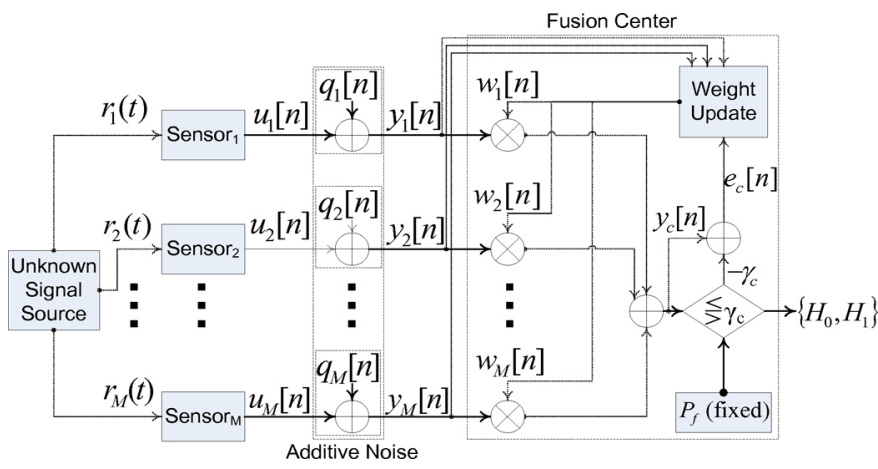

Fig. 1. System model for the proposed ADF algorithm.

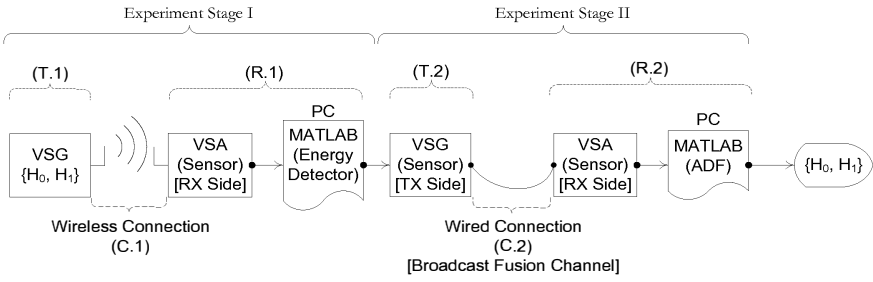

Fig. 2. Block diagram of the experimental setup emulating the system model given in Figure 1 along with the corresponding identifiers for different types of channels, transmitters, and receivers.

second case, the output of (C.2) is assumed to pass through a low-pass filter which is assumed to exist inherently at the receiver before (R.2) yields the baseband I/Q samples. The results for both cases are shown in Figure 5. As shown in the figure, low-pass filtering improves the performance of the $\mathrm{ADF}$ algorithm. This mainly stems from the fact that correlated inputs yield small error values in (19) which in turn results in fast convergence of weights in (20). It is important to state that such a correlation is already introduced into the data (as shown in Figure 3) to be fed into (T.2) since the same VSA is used throughout the experiment for both (R.1) and (R.2) in TDMA fashion.

\section{CONCLUSION}

An online adaptive cooperative spectrum sensing scheme based on POCS theory is proposed in order to maintain the performance and the reliability of spectrum sensing. Performance of the scheme is also investigated by a set of real data collected from an experimental setup. The contributions of this study are two-fold. First, an online adaptive data fusion scheme to be deployed in a cooperative spectrum sensing framework is introduced. Second, performance of the proposed method is validated through an experimental setup. Experimental results show that the proposed adaptive online cooperation method yields reliable spectrum sensing decisions based on probability of false alarm and detection metrics. Results also assure that the proposed spectrum sensing method can be utilized under challenging spectrum usage scenarios such as a cognitive radio system with a primary unknown signal source transmitting according to a Bernoulli trial process.

\section{ACKNOWLEDGMENT}

This work is supported by Qatar National Research Fund (QNRF) grant through National Priority Research Program 


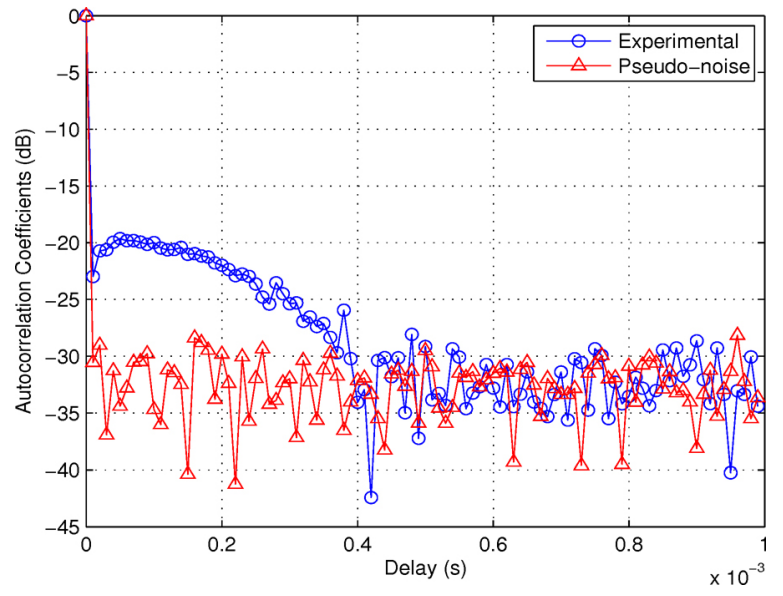

Fig. 3. Estimates of autocorrelation coefficients in logarithmic scale for experimental data corresponding to no-signal case $\left(H_{0}\right)$ versus computergenerated pseudo-noise. The impact of VSA filter manifests itself as an obvious correlation during the first $0.4 \mathrm{~ms}$.

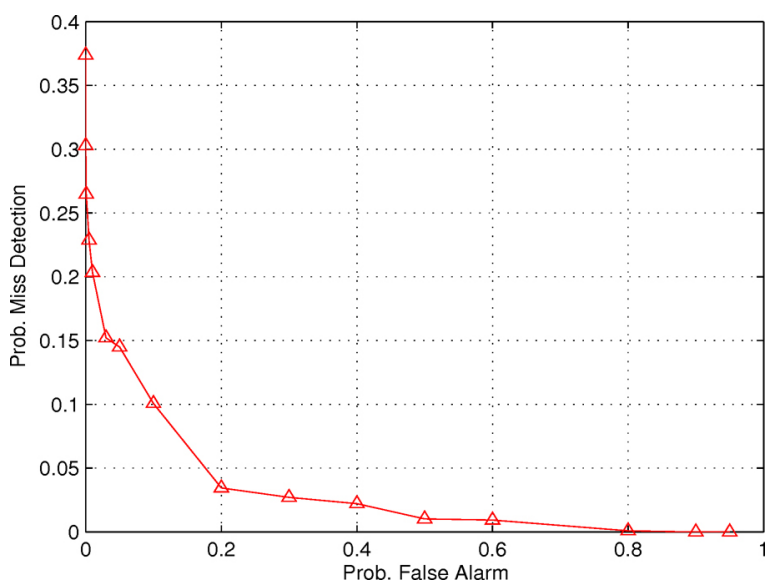

Fig. 4. The performance result of the proposed ADF mechanism for the experimental data collected. Here, note that $H_{0}$ and $H_{1}$ are selected according to a Bernoulli trial with a success rate of 0.5 , which implies maximum uncertainty about the absence/presence of the unknown signal source.

(NPRP) No. 08-101-2-025. QNRF is an initiative of Qatar Foundation. Authors would like to thank Wael Halbawi for his assistance during experiments.

\section{REFERENCES}

[1] J. Mitola, "Cognitive radio architecture evolution," Proceedings of the IEEE, vol. 97, no. 4, pp. 626-641, April 2009.

[2] Z. Quan, S. Cui, and A. H. Sayed, "Optimal linear cooperation for spectrum sensing in cognitive radio networks," IEEE J. Select. Topics in Sig. Processing, vol. 2, no. 1, pp. 28-40, Feb. 2008.

[3] K. B. Letaief and W. Zhang, "Cooperative communications for cognitive radio networks," Proceedings of the IEEE, vol. 97, no. 5, pp. 878-893, May 2009.

[4] K. A. Qaraqe and S. Yarkan, "A Second-Order Statistical Method for Spectrum Sensing in Correlated Shadowing and Fading Environments," in Proc. The 21st Annual IEEE International Symposium on Personal, Indoor and Mobile Radio Communications (PIMRC 2010), Istanbul, Türkiye, Sep. 26- 29, 2010, to appear.

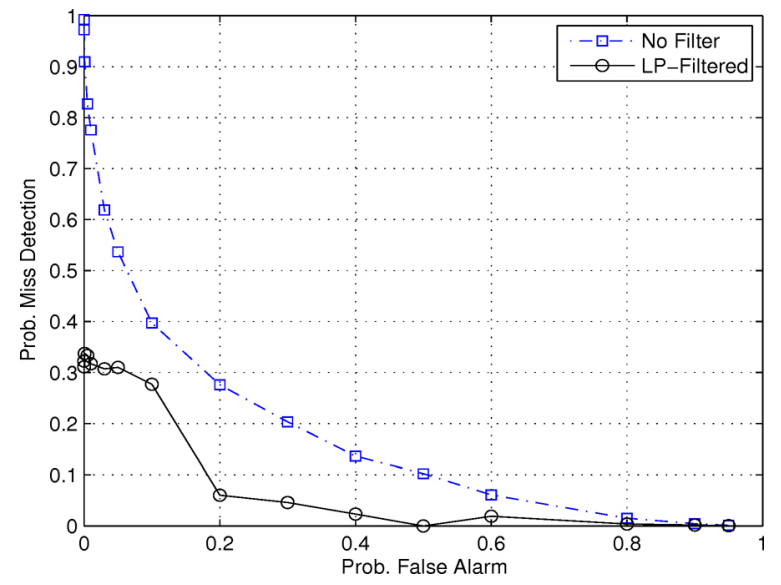

Fig. 5. The impact of filtering on the performance of the proposed ADF mechanism. Filtering occurs at the receiver (i.e., VSA) RF front-end due to practical design issues.

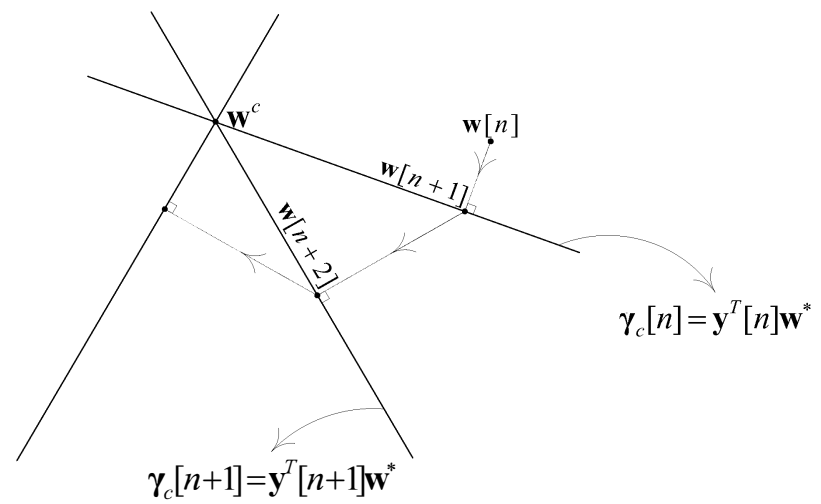

Fig. 6. Geometric interpretation: Weight vectors corresponding to correlator estimates at each time step are updated as to satisfy the hyperplane equations defined by the test threshold $\gamma_{c}[n]$ and the output of the broadcast fusion channel, $\mathbf{y}[n]$. Here, the lines represent hyperplanes in $\mathbb{R}^{M}$. Iterated weights converge to the intersection of hyperplanes, $\mathbf{w}^{\mathbf{c}}$, as discussed in [9].

[5] K. Zeng, P. Pawelczak, and D. Cabric, "Reputation-Based Cooperative Spectrum Sensing with Trusted Node Assistance," IEEE Communications Letters, vol. 14, no. 3, pp. 226-228, 2010.

[6] X. Zheng, J. Wang, Q. Wu, and J. Chen, "Cooperative spectrum sensing algorithm based on dempster-shafer theory," in 11th IEEE Singapore International Conference on Communication Systems (ICCS), 2008.

[7] Z. Quan, S. Cui, A. Sayed, and H. Poor, "Optimal multiband joint detection for spectrum sensing in cognitive radio networks," IEEE Transactions on Signal Processing, vol. 57, no. 3, pp. 1128-1140, 2009.

[8] B. Shen, K. Kwak, and Z. Bai, "Optimal linear soft fusion schemes for cooperative sensing in cognitive radio networks," in Proc. IEEE Global Telecommun. Conf. (GLOBECOM), 2009, pp. 1-6.

[9] P. L. Combettes, "The foundations of set theoretic estimation," Proceedings of the IEEE, vol. 81(2), pp. 182-208, 1993.

[10] D. C. Youla and H. Webb, "Image restoration by the method of convex projections, part i-theory," IEEE Trans. on Med. Imaging, vol. MI-I-2, pp. 81-94, 1982.

[11] U. Niesen, D. Shah, and G. W. Wornell, "Adaptive alternating minimization algorithms," IEEE Trans. Inform. Theory, vol. 55, no. 3, pp. 1423-1429, March 2009. 